\title{
Study of Routing Protocols on CBR and VBR Applications in VANET Scenario
}

\author{
Pooja Sharma \\ School of Computer Science \& Engg, \\ Shri Mata Vaishno Devi University Katra (J\&K)- 182320.India
}

\begin{abstract}
Vehicular Adhoc Networks (VANETs) are special type of Mobile Adhoc networks (MANETs) where node movement is in pre ordered fashion but with high velocity in comparison to MANETs where nodes move in random manner. Due to high mobility of nodes, reliable data streaming in vehicular networks is a complex and challenging task. Moreover, transmission of data is difficult because of varying requirements of different applications in terms of various resources like time, energy and bandwidth. This paper gives an overview of performance evaluation of four types of routing protocols on CBR and VBR applications. This paper emphasizes on packet delivery ratio, packet loss and packet loss ratio for CBR and VBR applications in different scenarios like varying node density, varying speed of nodes, pause times and packet size. The effectiveness of various routing protocols shows variation in different conditions. The performance evaluation of different applications in terms of Quality of service (QoS) parameters like packet delivery ratio, packet loss and packet loss ratio has been studied by varying different conditions of CBR traffic and VBR traffic which has gives an insight to improve packet delivery ratio which in turn can be utilized to improve performance of an application in future.
\end{abstract}

Keywords-VANETS; routing protocols; qualnet; traffic types introduction

\section{INTRODUCTION}

There are various applications like constant bit rate traffic (CBR), variable bit rate traffic (VBR), File Transfer protocol(FTP), Voice Over Internet Protocol(VOIP) . All these applications can be evaluated on various parameters in terms of throughput, jitter, average end to end delay. Internet traffic is broadly classified into two categories i.e real time applications or time critical traffic and the non real time applications or elastic applications. Real time traffic is further divided into two forms like real time streaming applications and real time control applications eg machine control, games. Examples of non real time applications are web browsing, email, FTP and telnet. Few of the real time and asymmetric applications are audio broadcasting, video broadcasting, interactive audio on demand, interactive video demand. Real time and symmetric applications are those which are conversant in nature such as teleconferencing (video and audio conferencing) and Voice over Internet protocol (VOIP).

There are various factors which affect the performance of different protocols along with applications like node density, velocity of nodes, mobility patterns, traffic types which will be implemented in this study. Effect of single hop routing and multi hop routing protocols can be easily observed in this research work. This research study basically analyzes the Quality of Service (QoS) metrics for different applications as mentioned on different protocols like AODV, DYMO, DSR and LAR so as to focus on some parameters where improvement can be done in order to optimize the performance of applications. This paper is divided into four different sections. First one is introduction followed by literature review and simulation and results and conclusion..

\section{LITERATURE REVIEW}

The existing literature has been reviewed regarding different requirements of various applications gathered from the various sources available. Some of the data has been taken from [1] and [2] regarding bandwidth, delay and jitter requirements for video streaming, VOIP, Interactive video etc. In continuous media, especially audio and video data has spatial relationships that must be taken care of. The requirements of time critical applications are commonly expressed as a set of values representing bandwidth, delay, and jitter and loss rate. Continuous streaming applications can cope with QoS which is significantly lower than real time streaming applications. Various voice encoding techniques such as G.711, G.726, G.727, G.728 are available, similarly several encoding or compression technique for $\mathrm{CD}$ quality sound have been developed such as MPEG Layer-1, MPEG Layer-2, MPEG Layer-3. The important building blocks of Prediction Based Routing ( PBR) protocol are obtaining location and velocity information of vehicles on the route to the gateway. Its basic operation is to create routes in the same way as reactive protocols. When a node wants to communicate, it sends route request (RREQ) packet with a TTL (time to live) value that specifies the number of hops to search for a gateway. In PBR, the lifetime of link between two nodes $i$ and $j$ is predicted as

Lifetime $_{\text {link }}=\frac{R-|d i j|}{\left|v_{i}-v_{j}\right|}$

where $\mathrm{R}$ is communication range of WLAN technology, $\left|\mathrm{d}_{\mathrm{ij}}\right|$ absolute distance between nodes $\mathrm{i}$ and $\mathrm{j}, \mathrm{v}_{\mathrm{i}}$ and $\mathrm{v}_{\mathrm{j}}$ are velocities.

Since a route comprises of one or more links, the route lifetime is the minimum of all its link lifetimes as done by Vinod Namboodri in [3]. With low vehicle density in the forward direction using routes through oncoming vehicles has an effect similar to doubling the vehicle density in the forward direction in terms of connectivity.

In one of the research study by shaily mittal [4] in "performance comparison of AODV, DSR and ZRP routing 
protocols in MANETs" analysis has been done for different routing protocols. One more research study has been done by Mrs. Vaishali D and Dr Ketan [5] in their study title "Simulation based performance evaluation of routing protocols in Vehicular Adhoc Networks". The above mentioned studies emphasize on effectiveness of different routing protocols in different situations.

Different protocols have different impact on throughput, jitter, delay and average end to end delay. Moreover, the type of application to be simulated matters a lot. There is a change observed in metrics for different applications like CBR applications, VBR applications, Telnet, FTP applications. Different applications in scenarios like varying node density, node speed, pause time, packet size are analyzed and results are observed keeping in consideration of vehicular adhoc networks.

\section{A. Adhoc on Demand Vector Routing Protocol (AODV)}

It is purely on -demand routing protocol. In order to find the path between source and destination a RREQ message is broadcasted to all the neighbor nodes who again continue to send messages to their neighbors until the destination is reached. Every node maintains two variables sequence number and broadcast ID in order to have loop free and maintain most recent information. Path discovery process and route maintenance is done in AODV as mentioned in [6] and [7].

\section{B. Dynamic Source Routing (DSR)}

Is also an on Demand routing protocol in which sequence of nodes through which packets will travel is calculated and information is stored in packet header. The source nodes sent request packets to all the neighbors in the network containing the address of the destination node and a reply is sent back to the source nodes with the list of network nodes through which it should move forward in the process. Route maintenance can be done either by hop by hop acknowledgement at the data link layer and end to end acknowledgements. Hop by Hop method allows early detection and retransmission of lost or corrupt packets in the data link layer. When the wireless transmission between two nodes do not work well then end to end replies on the application layer or transport layer may be used to indicate the status of the route from one host to the other. All intermediary nodes along the path simply forwards the packet to the next node as specified in the packet header[8].

\section{Location aided Routing (LAR)}

Protocol given by Young $\mathrm{Br}$ Ko et al [9] suggests an approach to utilize location information ( for instance , obtained by global positioning system) to improve performance of routing protocol for adhoc networks. By using location information, proposed protocol limits the search for a new route to a smaller request zone of the adhoc network. This results in significant reduction in number of messages. Location information is used for route discovery.

\section{Dynamic MANET on Demand Routing Protocol (DYMO)}

Enables dynamic, reactive, multi hop routing between participating nodes wishing to communicate. The basic operations of the protocol are route discovery and management. Using adhoc on demand distance vector (AODV), DYMO borrows "Path Accumulation" from Dynamic source routing and removes unnecessary route reply(RREP), precursor lists and Hello messages(Route exploration messages) thus simplifying AODV[10]. It retains sequence numbers and Route error messages from AODV [11]

The above mentioned protocols have been implemented on MANETs very well but their performance on VANETs is still not done as VANET nodes have high mobility which is covered in this paper. Moreover, these set of four protocols have been implemented on CBR and VBR applications to see that how these four protocols will satisfy most QoS parameters and show variations in packet delivery ratio, packet loss and packet loss ratio.

\section{SimULATION AND RESUlTS}

Qualnet version 5.0.1 is a discrete event simulator which is being used here. In this paper Qualnet is configured by having area $1500 \times 1500$ having different scenarios. Four protocols are taken into consideration i.e AODV,DSR,DYMO and LAR with varying network sizes, mobility of nodes by varying speeds of the nodes, pause time and vary size of packet ,simulation time. Different scenarios are made keeping some of the factors constant and change one at a time to see the impact of that particular factor on QoS metrics under observation. Very important, different traffic types will have different set of results keeping the same protocols when simulated in one of the scenario. In this study different formulae have been used for calculation of packet delivery ratio, packet loss and packet loss ratio.

\section{Perform}

ance Metrics

Packet Delivery Ratio is important metric to measure the performance of routing protocol .Packet Delivery Ratio is defined as the ratio of the total packets received by all destination nodes and the total packets sent by all source nodes[5].

Packet Delivery Ratio =

$\frac{\sum \quad \text { (Total packets received by all destination nodes }}{\sum \text { (Total packets send by all source nodes }}$

Packet Loss is the ratio of the number of packets that never reached the destination to the number of packets originated by the source. [5]

Mathematically, PL $=\frac{\text { nsentpackets-nreceivedpackets }}{\text { nsentpackets }}$
Packet Loss Ratio $=\frac{\text { nsentpackets-nreceivedpackets }}{\text { nsentpackets }} * 100$ 
TABLE I. VARIOUS PARAMETERS ARE USED WHILE VARYING NUMBER OF CONNECTIONS

\begin{tabular}{|l|l|}
\hline Parameter & Value \\
\hline Protocols & AODV,DSR,DYMO, LAR \\
\hline Number of Nodes & $30,50,80,120$ \\
\hline Pause Time & $30,60,100 \mathrm{~s}$ \\
\hline Simulation time & $30 \mathrm{~s}$ \\
\hline Traffic Type & CBR, VBR \\
\hline Transmission Range & $250 \mathrm{~m}$ \\
\hline Mobility Model & Random Way point Model \\
\hline Simulation Area & $1500 x 1500$ \\
\hline Node Speed & $0,10,20,60,90 \mathrm{~km} / \mathrm{h}$ \\
\hline Interface Type & Queue \\
\hline MAC Protocol & 802.11 Ext \\
\hline Packet Size & 512,1024 \\
\hline Radio Propagation Model & Two Ray Ground \\
\hline
\end{tabular}

Table 1 is giving details of all the parameters used while varying number of connections.In this paper four scenarios are simulated on the basis of which certain results are obtained and some outcomes and inferences are drawn. The effect of all the four protocols namely AODV,DSR,DYMO and LAR are studied on both type of traffic like constant bit rate traffic and variable bit rate traffic in terms of packet delivery ratio, packet loss and packet loss \%.

Scenario I CBR Traffic and VBR traffic with varying node density in terms of PDR, Packet Loss ( PL), Packet Loss \%

TABLE II. VARYING NODE DENSITY

\begin{tabular}{|c|c|c|c|c|c|c|c|c|c|c|c|c|}
\hline $\begin{array}{l}\text { No } \\
\text { de } \\
\text { Den } \\
\text { sity }\end{array}$ & 30 & $\begin{array}{l}3 \\
0\end{array}$ & 30 & 50 & $\begin{array}{l}5 \\
0\end{array}$ & 50 & 80 & $\begin{array}{l}8 \\
0\end{array}$ & 80 & $\begin{array}{l}12 \\
0\end{array}$ & $\begin{array}{l}12 \\
0\end{array}$ & $\begin{array}{l}12 \\
0\end{array}$ \\
\hline & $\begin{array}{l}\mathrm{P} \\
\mathrm{D} \\
\mathrm{R}\end{array}$ & $\begin{array}{l}\mathrm{P} \\
\mathrm{L}\end{array}$ & $\begin{array}{l}\text { PL } \\
\%\end{array}$ & $\begin{array}{l}\mathrm{P} \\
\mathrm{D} \\
\mathrm{R}\end{array}$ & $\begin{array}{l}P \\
L\end{array}$ & $\begin{array}{l}\text { PL } \\
\%\end{array}$ & $\begin{array}{l}\mathrm{P} \\
\mathrm{D} \\
\mathrm{R}\end{array}$ & $\begin{array}{l}\mathrm{P} \\
\mathrm{L}\end{array}$ & $\begin{array}{l}\text { PL } \\
\%\end{array}$ & $\begin{array}{l}\mathrm{P} \\
\mathrm{D} \\
\mathrm{R}\end{array}$ & PL & $\begin{array}{l}\text { PL } \\
\%\end{array}$ \\
\hline $\begin{array}{l}\mathrm{AO} \\
\mathrm{DV}\end{array}$ & $\begin{array}{l}0 . \\
82\end{array}$ & $\begin{array}{l}0 . \\
1 \\
9\end{array}$ & $\begin{array}{l}19 \\
.1 \\
7\end{array}$ & $\begin{array}{l}0 . \\
83\end{array}$ & $\begin{array}{l}0 . \\
1 \\
8\end{array}$ & $\begin{array}{l}17 \\
.5 \\
0\end{array}$ & $\begin{array}{l}0 . \\
81\end{array}$ & $\begin{array}{l}0 . \\
1 \\
9\end{array}$ & $\begin{array}{l}19 \\
.1 \\
7\end{array}$ & $\begin{array}{l}0 . \\
87 \\
5\end{array}$ & $\begin{array}{l}0 . \\
12 \\
5\end{array}$ & $\begin{array}{l}12 . \\
50 \\
0\end{array}$ \\
\hline $\begin{array}{l}\mathrm{DS} \\
\mathrm{R}\end{array}$ & $\begin{array}{l}0 . \\
64\end{array}$ & $\begin{array}{l}0 . \\
3 \\
6\end{array}$ & $\begin{array}{l}35 \\
.8 \\
3\end{array}$ & $\begin{array}{l}0 . \\
65\end{array}$ & $\begin{array}{l}0 . \\
3 \\
5\end{array}$ & $\begin{array}{l}35 \\
.0 \\
0\end{array}$ & $\begin{array}{l}0 . \\
66\end{array}$ & $\begin{array}{l}0 . \\
3 \\
4\end{array}$ & $\begin{array}{l}34 \\
.1 \\
7\end{array}$ & $\begin{array}{l}0 . \\
67 \\
5\end{array}$ & $\begin{array}{l}0 . \\
32 \\
5\end{array}$ & $\begin{array}{l}32 . \\
50 \\
0\end{array}$ \\
\hline $\begin{array}{l}\text { DY } \\
\text { MO }\end{array}$ & $\begin{array}{l}0 . \\
83\end{array}$ & $\begin{array}{l}0 . \\
1 \\
7\end{array}$ & $\begin{array}{l}16 \\
.6 \\
7\end{array}$ & $\begin{array}{l}0 . \\
78\end{array}$ & $\begin{array}{l}0 . \\
2 \\
2\end{array}$ & $\begin{array}{l}21 \\
.6 \\
7\end{array}$ & $\begin{array}{l}0 . \\
78\end{array}$ & $\begin{array}{l}0 . \\
2 \\
3\end{array}$ & $\begin{array}{l}22 \\
.5 \\
0\end{array}$ & $\begin{array}{l}0 . \\
85 \\
8\end{array}$ & $\begin{array}{l}0 . \\
14 \\
2\end{array}$ & $\begin{array}{l}14 . \\
16 \\
7\end{array}$ \\
\hline $\begin{array}{l}\text { LA } \\
\text { R }\end{array}$ & $\begin{array}{l}0 . \\
88\end{array}$ & $\begin{array}{l}0 . \\
1 \\
2\end{array}$ & $\begin{array}{l}11 \\
.6 \\
7\end{array}$ & $\begin{array}{l}0 . \\
88\end{array}$ & $\begin{array}{l}0 . \\
1 \\
3\end{array}$ & $\begin{array}{l}12 \\
.5 \\
0\end{array}$ & $\begin{array}{l}0 . \\
89\end{array}$ & $\begin{array}{l}0 . \\
1 \\
1\end{array}$ & $\begin{array}{l}10 \\
.8 \\
3\end{array}$ & $\begin{array}{l}0 . \\
85 \\
8\end{array}$ & $\begin{array}{l}0 . \\
14 \\
2\end{array}$ & $\begin{array}{l}14 . \\
16 \\
7\end{array}$ \\
\hline
\end{tabular}

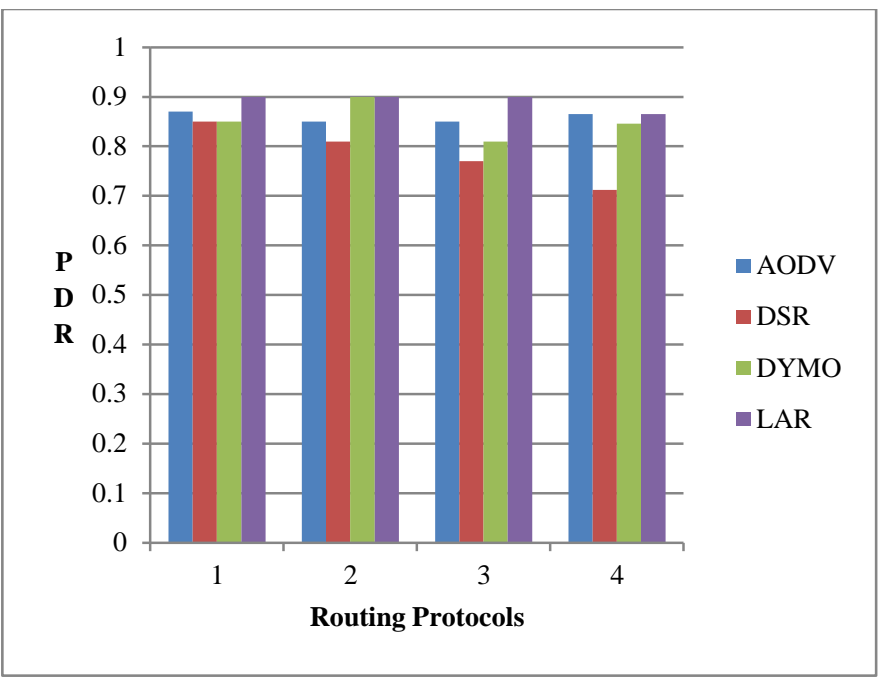

Fig. 1. Packet Delivery Ratio for CBR Traffic with varying Node Density

TABLE III. VBR TRAFFIC WITH VARYING NODE DENSITY

\begin{tabular}{|c|c|c|c|c|c|c|c|c|c|c|c|c|}
\hline $\begin{array}{l}\text { No } \\
\text { de } \\
\text { Den } \\
\text { sity }\end{array}$ & 30 & $\begin{array}{l}3 \\
0\end{array}$ & 30 & 50 & $\begin{array}{l}5 \\
0\end{array}$ & 50 & 80 & $\begin{array}{l}8 \\
0\end{array}$ & 80 & $\begin{array}{l}12 \\
0\end{array}$ & $\begin{array}{l}12 \\
0\end{array}$ & $\begin{array}{l}12 \\
0\end{array}$ \\
\hline & $\begin{array}{l}\mathrm{P} \\
\mathrm{D} \\
\mathrm{R}\end{array}$ & $\begin{array}{l}\mathrm{P} \\
\mathrm{L}\end{array}$ & $\begin{array}{l}\text { PL } \\
\%\end{array}$ & $\begin{array}{l}\mathrm{P} \\
\mathrm{D} \\
\mathrm{R}\end{array}$ & $\begin{array}{l}\mathrm{P} \\
\mathrm{L}\end{array}$ & $\begin{array}{l}\text { PL } \\
\%\end{array}$ & $\begin{array}{l}\mathrm{P} \\
\mathrm{D} \\
\mathrm{R}\end{array}$ & $\begin{array}{l}\mathrm{P} \\
\mathrm{L}\end{array}$ & $\begin{array}{l}\text { PL } \\
\%\end{array}$ & $\begin{array}{l}\mathrm{P} \\
\mathrm{D} \\
\mathrm{R}\end{array}$ & PL & $\begin{array}{l}\text { PL } \\
\%\end{array}$ \\
\hline $\begin{array}{l}\mathrm{AO} \\
\mathrm{DV}\end{array}$ & $\begin{array}{l}0 . \\
87\end{array}$ & $\begin{array}{l}0 . \\
1 \\
3\end{array}$ & $\begin{array}{l}13 \\
.4 \\
6\end{array}$ & $\begin{array}{l}0 . \\
85\end{array}$ & $\begin{array}{l}0 . \\
1 \\
5\end{array}$ & $\begin{array}{l}15 \\
.3 \\
8\end{array}$ & $\begin{array}{l}0 . \\
85\end{array}$ & $\begin{array}{l}0 . \\
1 \\
5\end{array}$ & $\begin{array}{l}15 \\
.3 \\
8\end{array}$ & $\begin{array}{l}0 . \\
86 \\
5\end{array}$ & $\begin{array}{l}0 . \\
13 \\
5\end{array}$ & $\begin{array}{l}13 . \\
46 \\
2\end{array}$ \\
\hline $\begin{array}{l}\mathrm{DS} \\
\mathrm{R}\end{array}$ & $\begin{array}{l}0 . \\
85\end{array}$ & $\begin{array}{l}0 . \\
1 \\
5\end{array}$ & $\begin{array}{l}15 \\
.3 \\
8 \\
\end{array}$ & $\begin{array}{l}0 . \\
81\end{array}$ & $\begin{array}{l}0 . \\
1 \\
9\end{array}$ & $\begin{array}{l}19 \\
.2 \\
3 \\
\end{array}$ & $\begin{array}{l}0 . \\
77\end{array}$ & $\begin{array}{l}0 . \\
2 \\
3\end{array}$ & $\begin{array}{l}23 \\
.0 \\
8 \\
\end{array}$ & $\begin{array}{l}0 . \\
71 \\
2 \\
\end{array}$ & $\begin{array}{l}0 . \\
28 \\
8 \\
\end{array}$ & $\begin{array}{l}28 . \\
84 \\
6 \\
\end{array}$ \\
\hline $\begin{array}{l}\text { DY } \\
\text { MO }\end{array}$ & $\begin{array}{l}0 . \\
85\end{array}$ & $\begin{array}{l}0 . \\
1 \\
5\end{array}$ & $\begin{array}{l}15 \\
.3 \\
8\end{array}$ & $\begin{array}{l}0 . \\
90\end{array}$ & $\begin{array}{l}0 . \\
1 \\
0\end{array}$ & $\begin{array}{l}9 . \\
62\end{array}$ & $\begin{array}{l}0 . \\
81\end{array}$ & $\begin{array}{l}0 . \\
1 \\
9\end{array}$ & $\begin{array}{l}19 \\
.2 \\
3\end{array}$ & $\begin{array}{l}0 . \\
84 \\
6\end{array}$ & $\begin{array}{l}0 . \\
15 \\
4\end{array}$ & $\begin{array}{l}15 . \\
38 \\
5\end{array}$ \\
\hline $\begin{array}{l}\text { LA } \\
\text { R }\end{array}$ & $\begin{array}{l}0 . \\
90\end{array}$ & $\begin{array}{l}0 . \\
1 \\
0\end{array}$ & $\begin{array}{l}9 . \\
62\end{array}$ & $\begin{array}{l}0 . \\
90\end{array}$ & $\begin{array}{l}0 . \\
1 \\
0\end{array}$ & $\begin{array}{l}9 . \\
62\end{array}$ & $\begin{array}{l}0 . \\
90\end{array}$ & $\begin{array}{l}0 . \\
1 \\
0\end{array}$ & $\begin{array}{l}9 . \\
62\end{array}$ & $\begin{array}{l}0 . \\
86 \\
5\end{array}$ & $\begin{array}{l}0 . \\
13 \\
5\end{array}$ & $\begin{array}{l}13 . \\
46 \\
2\end{array}$ \\
\hline
\end{tabular}

It is observed here in table 2 and table 3 that Packet Delivery Ratio has been decreasing with increasing node density truly for the VBR traffic for AODV, DSR, DYMO whereas not much effect has been seen for LAR with increasing node density. For DYMO, there is no fixed pattern observed for packet delivery ratio for VBR traffic. We can observe in Figure 1 and Figure 2,CBR traffic, AODV and DSR there has been reduction in packet delivery ratio but when nodes are increased to 120, a gain in PDR has been observed. Similar is the case for DYMO but LAR keeps a steady pace for packet delivery ratio in turn keeping packet loss ratio more or less same. 


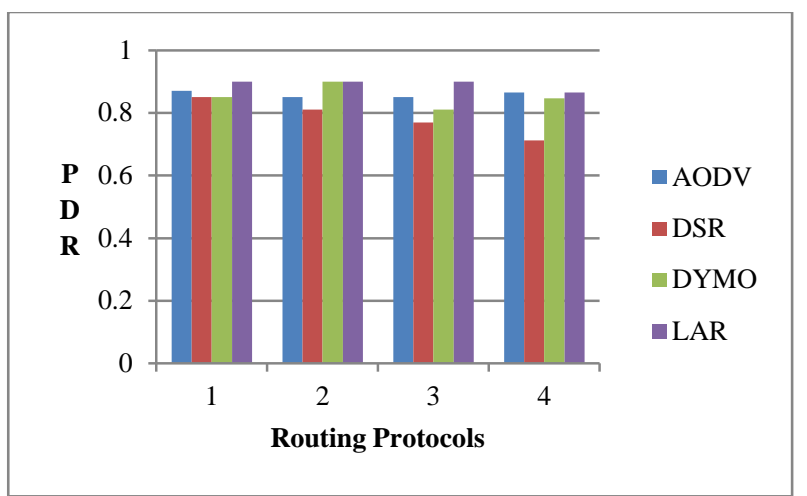

Fig. 2. Packet Delivery Ratio for VBR Traffic with varying node density

Scenario II Node Density Fixed, speed of the nodes varying CBR (speed in mps)

TABLE IV. EFFECT OF MOBILITY OF NODES ON CBR TRAFFIC IN TERMS OF PDR, PL, PL RATIO

\begin{tabular}{|l|l|l|l|l|l|l|l|l|l|}
\hline $\begin{array}{l}\text { Node } \\
\text { Speed }\end{array}$ & $\begin{array}{l}10- \\
20\end{array}$ & $\begin{array}{l}10- \\
20\end{array}$ & $\begin{array}{l}10- \\
20\end{array}$ & $\begin{array}{l}20- \\
60\end{array}$ & $\begin{array}{l}20- \\
60\end{array}$ & $\begin{array}{l}20- \\
60\end{array}$ & $\begin{array}{l}20- \\
90\end{array}$ & $\begin{array}{l}20- \\
90\end{array}$ & $\begin{array}{l}20- \\
90\end{array}$ \\
\hline R & PL & $\begin{array}{l}\text { PL } \\
\%\end{array}$ & $\begin{array}{l}\text { PD } \\
\text { R }\end{array}$ & PL & PL\% & $\begin{array}{l}\text { PD } \\
\text { R }\end{array}$ & PL & PL\% \\
\hline $\begin{array}{l}\text { AOD } \\
\text { V }\end{array}$ & 0.92 & $\begin{array}{l}0.0 \\
8\end{array}$ & 8.33 & 0.81 & $\begin{array}{l}0.1 \\
9\end{array}$ & $\begin{array}{l}19.1 \\
7\end{array}$ & 0.82 & $\begin{array}{l}0.1 \\
8\end{array}$ & $\begin{array}{l}18.3 \\
3\end{array}$ \\
\hline DSR & 0.94 & $\begin{array}{l}0.0 \\
6\end{array}$ & 5.83 & 0.64 & $\begin{array}{l}0.3 \\
6\end{array}$ & $\begin{array}{l}35.8 \\
3\end{array}$ & 0.69 & $\begin{array}{l}0.3 \\
1\end{array}$ & $\begin{array}{l}30.8 \\
3\end{array}$ \\
\hline $\begin{array}{l}\text { DYM } \\
\text { O }\end{array}$ & 0.92 & $\begin{array}{l}0.0 \\
8\end{array}$ & 8.33 & 0.83 & $\begin{array}{l}0.1 \\
7\end{array}$ & $\begin{array}{l}16.6 \\
7\end{array}$ & 0.86 & $\begin{array}{l}0.1 \\
4\end{array}$ & $\begin{array}{l}14.1 \\
7\end{array}$ \\
\hline LAR & 0.93 & $\begin{array}{l}0.0 \\
7\end{array}$ & 6.67 & 0.88 & $\begin{array}{l}0.1 \\
2\end{array}$ & $\begin{array}{l}11.6 \\
7\end{array}$ & 0.88 & $\begin{array}{l}0.1 \\
3\end{array}$ & $\begin{array}{l}12.5 \\
0\end{array}$ \\
\hline
\end{tabular}

TABLE V. EFFECT OF MOBILITy OF NODES ON VBR TRAFFiC IN TERMS OF PDR, PL, PL RATIO (SPEED IN MPS)

\begin{tabular}{|l|l|l|l|l|l|l|l|l|l|}
\hline $\begin{array}{l}\text { Node } \\
\text { Speed }\end{array}$ & $\begin{array}{l}10- \\
20\end{array}$ & $\begin{array}{l}10- \\
20\end{array}$ & $\begin{array}{l}10- \\
20\end{array}$ & $\begin{array}{l}20- \\
60\end{array}$ & $\begin{array}{l}20- \\
60\end{array}$ & $\begin{array}{l}20- \\
60\end{array}$ & $\begin{array}{l}20- \\
90\end{array}$ & $\begin{array}{l}20- \\
90\end{array}$ & $\begin{array}{l}20- \\
90\end{array}$ \\
\hline R & PL & $\begin{array}{l}\text { PL } \\
\%\end{array}$ & $\begin{array}{l}\text { PD } \\
\text { R }\end{array}$ & PL & PL\% & $\begin{array}{l}\text { PD } \\
\text { R }\end{array}$ & PL & PL\% \\
\hline $\begin{array}{l}\text { AOD } \\
\text { V }\end{array}$ & 0.92 & $\begin{array}{l}0.0 \\
8\end{array}$ & 7.69 & 0.87 & $\begin{array}{l}0.1 \\
3\end{array}$ & $\begin{array}{l}13.4 \\
6\end{array}$ & 0.81 & $\begin{array}{l}0.1 \\
9\end{array}$ & $\begin{array}{l}19.2 \\
3\end{array}$ \\
\hline DSR & 0.96 & $\begin{array}{l}0.0 \\
4\end{array}$ & 3.85 & 0.85 & $\begin{array}{l}0.1 \\
5\end{array}$ & $\begin{array}{l}15.3 \\
8\end{array}$ & 0.77 & $\begin{array}{l}0.2 \\
3\end{array}$ & $\begin{array}{l}23.0 \\
8\end{array}$ \\
\hline $\begin{array}{l}\text { DYM } \\
\text { O }\end{array}$ & 0.90 & $\begin{array}{l}0.1 \\
0\end{array}$ & 9.62 & 0.85 & $\begin{array}{l}0.1 \\
5\end{array}$ & $\begin{array}{l}15.3 \\
8\end{array}$ & 0.85 & $\begin{array}{l}0.1 \\
5\end{array}$ & $\begin{array}{l}15.3 \\
8\end{array}$ \\
\hline LAR & 0.92 & $\begin{array}{l}0.0 \\
8\end{array}$ & 7.69 & 0.90 & $\begin{array}{l}0.1 \\
0\end{array}$ & 9.62 & 0.87 & $\begin{array}{l}0.1 \\
3\end{array}$ & $\begin{array}{l}13.4 \\
6\end{array}$ \\
\hline
\end{tabular}

As observed in Table 4 and 5, Packet delivery ratio has been perfectly decreasing with the increasing velocity of nodes for VBR traffic for all the four protocols but CBR traffic is showing irregularities in its behavior in terms of packet delivery ratio. DSR is hugely reducing packet delivery ratio when increasing speed of nodes for CBR traffic whereas AODV, LAR and DYMO are more or less behaving in same manner. In figure 3 and 4 , it is observed that LAR is giving maximum packet delivery ratio even with varying and highest speed of minimum 20 and maximum 90 mps.

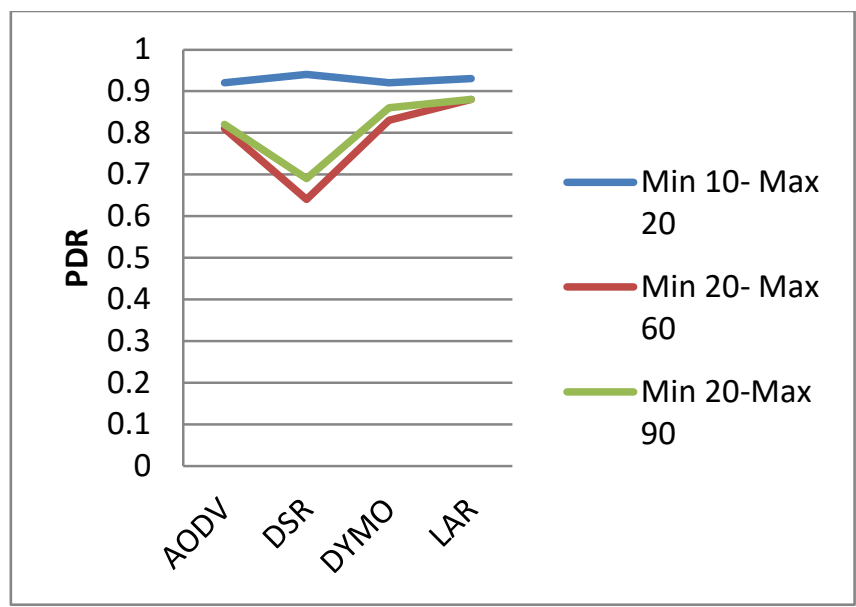

Fig. 3. Packet delivery ratio for CBR traffic with varying speed of nodes

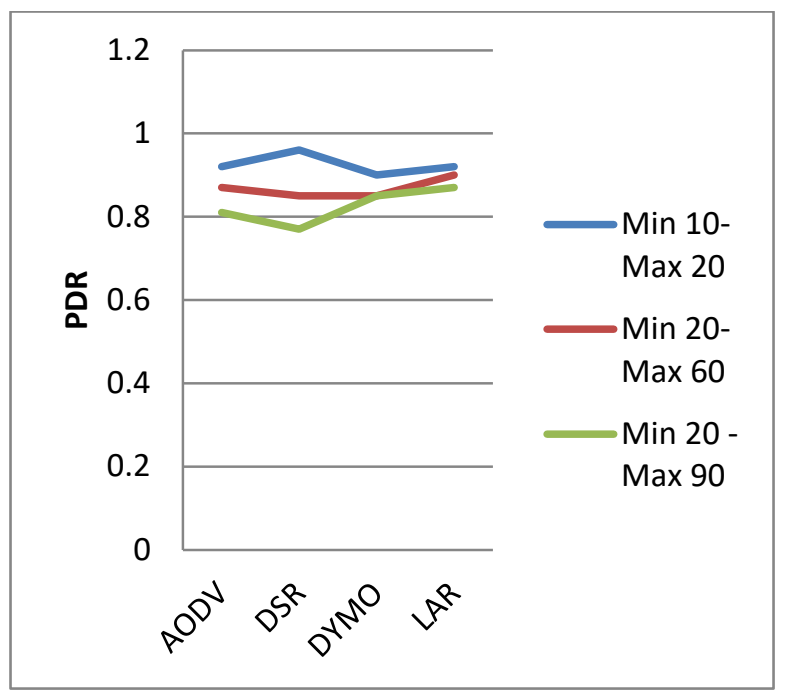

Fig. 4. Packet Delivery Ratio of VBR Traffic with varying speed of nodes

\section{Scenario III Varying pause time on CBR and VBR Traffic}

TABLE VI. CBR TRAFFic With DifFERENT PAUSE TIMES

\begin{tabular}{|l|l|l|l|l|l|l|l|l|l|}
\hline $\begin{array}{l}\text { Pause } \\
\text { Time }\end{array}$ & $30 \mathrm{~s}$ & $30 \mathrm{~s}$ & $30 \mathrm{~s}$ & $60 \mathrm{~s}$ & $60 \mathrm{~s}$ & $60 \mathrm{~s}$ & $\begin{array}{l}100 \\
\mathrm{~s}\end{array}$ & $\begin{array}{l}100 \\
\mathrm{~s}\end{array}$ & $100 \mathrm{~s}$ \\
\hline $\begin{array}{l}\mathrm{PD} \\
\mathrm{R}\end{array}$ & $\mathrm{PL}$ & $\begin{array}{l}\mathrm{PL} \\
\%\end{array}$ & $\begin{array}{l}\text { PD } \\
\mathrm{R}\end{array}$ & PL & PL\% & $\begin{array}{l}\text { PD } \\
\mathrm{R}\end{array}$ & PL & PL\% \\
\hline $\begin{array}{l}\text { AOD } \\
\text { V }\end{array}$ & 0.92 & $\begin{array}{l}0.0 \\
8\end{array}$ & 8.33 & 0.90 & $\begin{array}{l}0.1 \\
0\end{array}$ & $\begin{array}{l}10.0 \\
0\end{array}$ & 0.88 & 0.12 & $\begin{array}{l}11.6 \\
7\end{array}$ \\
\hline DSR & 0.94 & $\begin{array}{l}0.0 \\
6\end{array}$ & 5.83 & 0.95 & $\begin{array}{l}0.0 \\
5\end{array}$ & 5.00 & 0.93 & 0.08 & 7.50 \\
\hline $\begin{array}{l}\text { DYM } \\
\text { O }\end{array}$ & 0.92 & $\begin{array}{l}0.0 \\
8\end{array}$ & 8.33 & 0.93 & $\begin{array}{l}0.0 \\
8\end{array}$ & 7.50 & 0.92 & 0.08 & 8.33 \\
\hline LAR & 0.93 & $\begin{array}{l}0.0 \\
7\end{array}$ & 6.67 & 0.83 & $\begin{array}{l}0.1 \\
7\end{array}$ & $\begin{array}{l}16.6 \\
7\end{array}$ & 0.96 & 0.04 & 4.17 \\
\hline
\end{tabular}




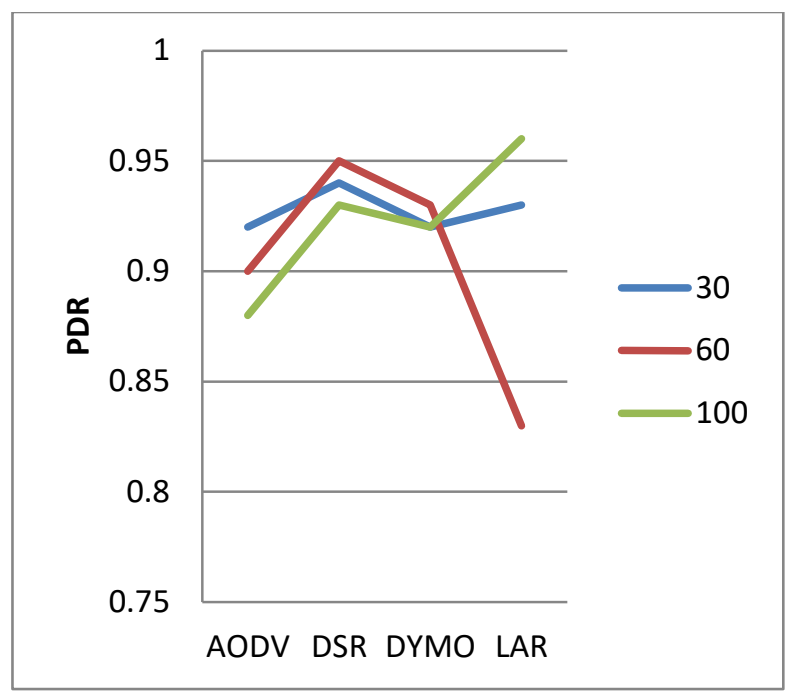

Fig. 5. Effect of changing pause times on CBR in terms of packet delivery ratio

TABLE VII. VBR TRAFFIC With CHANGING PAUSE TIMES IN TERMS OF PDR, PL, PACKET LOSS \%

\begin{tabular}{|c|c|c|c|c|c|c|c|c|c|}
\hline $\begin{array}{l}\text { Pause } \\
\text { Time }\end{array}$ & $30 \mathrm{~s}$ & $30 \mathrm{~s}$ & $30 \mathrm{~s}$ & $60 \mathrm{~s}$ & $60 \mathrm{~s}$ & $60 \mathrm{~s}$ & $\begin{array}{l}100 \\
\mathrm{~s}\end{array}$ & $\begin{array}{l}100 \\
\mathrm{~s}\end{array}$ & $100 \mathrm{~s}$ \\
\hline & $\begin{array}{l}\mathrm{PD} \\
\mathrm{R}\end{array}$ & PL & $\begin{array}{l}\text { PL } \\
\%\end{array}$ & $\begin{array}{l}\mathrm{PD} \\
\mathrm{R}\end{array}$ & PL & $\begin{array}{l}\text { PL } \\
\%\end{array}$ & $\begin{array}{l}\mathrm{PD} \\
\mathrm{R}\end{array}$ & PL & PL\% \\
\hline $\begin{array}{l}\text { AOD } \\
\mathrm{V}\end{array}$ & 0.92 & $\begin{array}{l}0.0 \\
8\end{array}$ & 7.69 & 0.98 & $\begin{array}{l}0.0 \\
2\end{array}$ & 1.92 & 0.83 & 0.17 & $\begin{array}{l}17.3 \\
1\end{array}$ \\
\hline DSR & 0.96 & $\begin{array}{l}0.0 \\
4\end{array}$ & 3.85 & 0.96 & $\begin{array}{l}0.0 \\
4\end{array}$ & 3.85 & 0.94 & 0.06 & 5.77 \\
\hline $\begin{array}{l}\text { DYM } \\
\text { O }\end{array}$ & 0.90 & $\begin{array}{l}0.1 \\
0\end{array}$ & 9.62 & 0.94 & $\begin{array}{l}0.0 \\
6\end{array}$ & 5.77 & 0.92 & 0.08 & 7.69 \\
\hline LAR & 0.92 & $\begin{array}{l}0.0 \\
8\end{array}$ & 7.69 & 0.94 & $\begin{array}{l}0.0 \\
6\end{array}$ & 5.77 & 0.96 & 0.04 & 3.85 \\
\hline
\end{tabular}

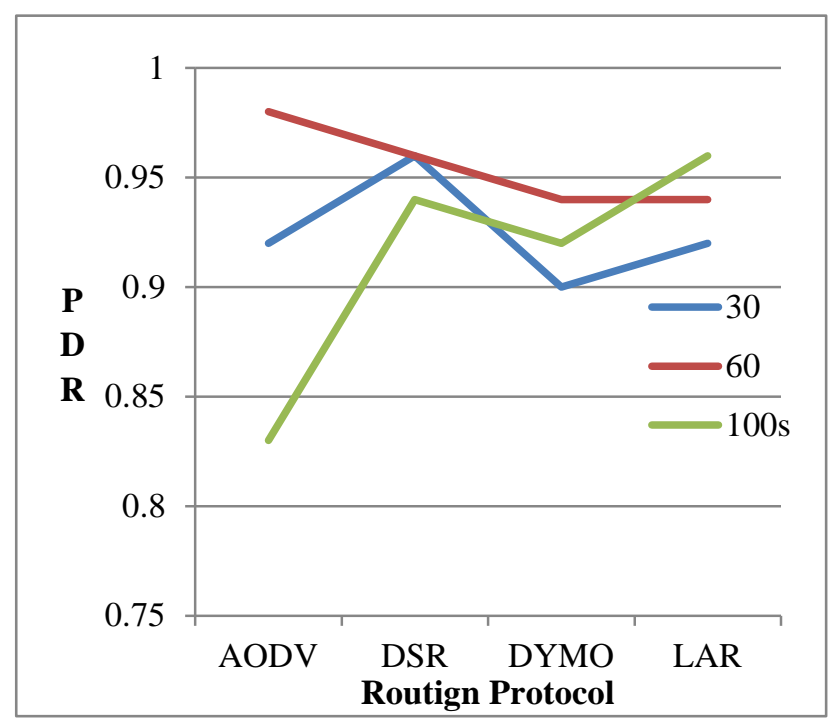

Fig. 6. VBR Traffic with changing pause times in terms of packet delivery Ratio
When pause times are varied, as seen in Figure 5 and 6 along with table 6 and 7,significant decrease has been seen in packet delivery ratio for CBR traffic especially when pause time is made 60s whereas for pause time 100s, there has been reduction in packet delivery ratio for AODV and DSR. DYMO doesn't change packet delivery ratio but LAR increases PDR. For VBR traffic, there has been an increase in PDR for all the protocols except DSR which is not making any change in PDR when pause time is 60s but when talking of 100s, there has been an decrease in PDR for AODV and DSR but DYMO and LAR increases PDR and so reduces packet loss ratio.

Scenario IV Vary packet size of the traffic for CBR and VBR

TABLE VIII. EFFECT OF PROTOCOLS ON CBR AND VBR IN TERMS OF PDR, PL, PL\%

\begin{tabular}{|c|c|c|c|c|c|c|c|c|c|c|c|c|}
\hline $\begin{array}{l}\text { Pac } \\
\text { ket } \\
\text { Siz } \\
\text { e }\end{array}$ & $\begin{array}{l}51 \\
2\end{array}$ & $\begin{array}{l}5 \\
1 \\
2\end{array}$ & $\begin{array}{l}51 \\
2\end{array}$ & $\begin{array}{l}10 \\
24\end{array}$ & $\begin{array}{l}10 \\
24\end{array}$ & $\begin{array}{l}10 \\
24\end{array}$ & $\begin{array}{l}51 \\
2\end{array}$ & $\begin{array}{l}5 \\
1 \\
2\end{array}$ & $\begin{array}{l}51 \\
2\end{array}$ & $\begin{array}{l}10 \\
24\end{array}$ & $\begin{array}{l}10 \\
24\end{array}$ & $\begin{array}{l}10 \\
24\end{array}$ \\
\hline & \multicolumn{6}{|c|}{ CBR } & \multicolumn{6}{|c|}{ VBR } \\
\hline & $\begin{array}{l}\mathrm{P} \\
\mathrm{D} \\
\mathrm{R}\end{array}$ & $\begin{array}{l}\mathrm{P} \\
\mathrm{L}\end{array}$ & $\begin{array}{l}\mathrm{PL} \\
\%\end{array}$ & $\begin{array}{l}\mathrm{P} \\
\mathrm{D} \\
\mathrm{R}\end{array}$ & $\begin{array}{l}\mathrm{P} \\
\mathrm{L}\end{array}$ & $\begin{array}{l}\mathrm{PL} \\
\%\end{array}$ & $\begin{array}{l}\mathrm{P} \\
\mathrm{D} \\
\mathrm{R}\end{array}$ & $\begin{array}{l}\mathrm{P} \\
\mathrm{L}\end{array}$ & $\begin{array}{l}\mathrm{PL} \\
\%\end{array}$ & $\begin{array}{l}\mathrm{P} \\
\mathrm{D} \\
\mathrm{R}\end{array}$ & $\begin{array}{l}\mathrm{P} \\
\mathrm{L}\end{array}$ & $\begin{array}{l}\mathrm{PL} \\
\%\end{array}$ \\
\hline $\begin{array}{l}\mathrm{AO} \\
\mathrm{DV}\end{array}$ & $\begin{array}{l}0 . \\
81\end{array}$ & $\begin{array}{l}0 . \\
1 \\
9\end{array}$ & $\begin{array}{l}19 . \\
17\end{array}$ & $\begin{array}{l}0 . \\
79\end{array}$ & $\begin{array}{l}0 . \\
21\end{array}$ & $\begin{array}{l}20 . \\
83\end{array}$ & $\begin{array}{l}0 . \\
87\end{array}$ & $\begin{array}{l}0 . \\
1 \\
3\end{array}$ & $\begin{array}{l}13 \\
46\end{array}$ & $\begin{array}{l}0 . \\
88\end{array}$ & $\begin{array}{l}0 . \\
12\end{array}$ & $\begin{array}{l}11 \\
54\end{array}$ \\
\hline $\begin{array}{l}\mathrm{DS} \\
\mathrm{R}\end{array}$ & $\begin{array}{l}0 . \\
64\end{array}$ & $\begin{array}{l}0 . \\
3 \\
6\end{array}$ & $\begin{array}{l}35 . \\
83\end{array}$ & $\begin{array}{l}0 . \\
73\end{array}$ & $\begin{array}{l}0 . \\
28\end{array}$ & $\begin{array}{l}27 . \\
50\end{array}$ & $\begin{array}{l}0 . \\
85\end{array}$ & $\begin{array}{l}0 . \\
1 \\
5\end{array}$ & $\begin{array}{l}15 . \\
38\end{array}$ & $\begin{array}{l}0 . \\
90\end{array}$ & $\begin{array}{l}0 . \\
10\end{array}$ & $\begin{array}{l}9.6 \\
2\end{array}$ \\
\hline $\begin{array}{l}\text { DY } \\
\text { MO }\end{array}$ & $\begin{array}{l}0 . \\
83\end{array}$ & $\begin{array}{l}0 . \\
1 \\
7\end{array}$ & $\begin{array}{l}16 . \\
67\end{array}$ & $\begin{array}{l}0 . \\
71\end{array}$ & $\begin{array}{l}0 . \\
29\end{array}$ & $\begin{array}{l}29 . \\
17\end{array}$ & $\begin{array}{l}0 . \\
85\end{array}$ & $\begin{array}{l}0 . \\
1 \\
5\end{array}$ & $\begin{array}{l}15 \\
38\end{array}$ & $\begin{array}{l}0 . \\
79\end{array}$ & $\begin{array}{l}0 . \\
21\end{array}$ & $\begin{array}{l}21 \\
15\end{array}$ \\
\hline $\begin{array}{l}\text { LA } \\
\text { R }\end{array}$ & $\begin{array}{l}0 . \\
88\end{array}$ & $\begin{array}{l}0 . \\
1 \\
2\end{array}$ & $\begin{array}{l}11 . \\
67\end{array}$ & $\begin{array}{l}0 . \\
88\end{array}$ & $\begin{array}{l}0 . \\
13\end{array}$ & $\begin{array}{l}12 . \\
50\end{array}$ & $\begin{array}{l}0 . \\
90\end{array}$ & $\begin{array}{l}0 . \\
1 \\
0\end{array}$ & $\begin{array}{l}9.6 \\
2\end{array}$ & $\begin{array}{l}0 . \\
81\end{array}$ & $\begin{array}{l}0 . \\
19\end{array}$ & $\begin{array}{l}19 . \\
23\end{array}$ \\
\hline
\end{tabular}

Figure 7 is giving an information as Packet size is varied in fourth scenario by making 1024 bytes for CBR and VBR traffic instead of 524 bytes. The impact of four protocols has been studied like AODV, DSR, DYMO and LAR. In table 8,There has been an articulate decrease seen in packet delivery ratio for all protocols except DSR where an increase is seen for CBR traffic. As far as VBR traffic is concerned, there has been an increase in packet delivery ratio for AODV and DSR, but for DYMO and LAR there has been a decrease in packet delivery ratio 


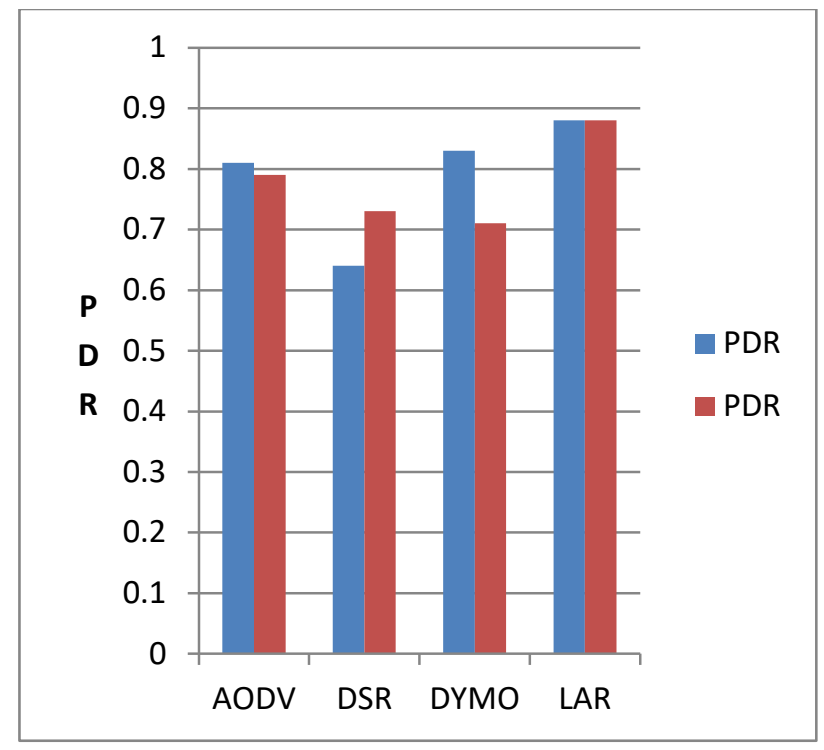

(a)

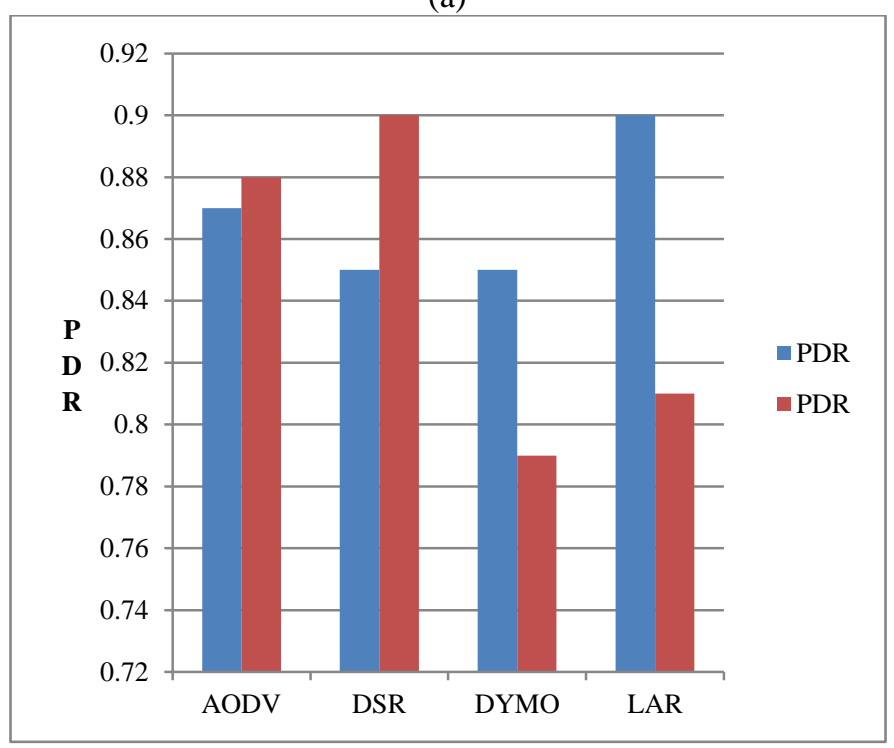

(b)

Fig. 7. CBR Traffic showing PDR with varying packet size for four protocols

\section{CONCLUSION}

The above qualitative results show that AODV has been found to be better in most of the scenarios followed by LAR. AODV and LAR have shown showing little variations in PDR with reference to node density whereas DSR has shown large variation in PDR. With reference to the change in mobility of nodes, all the four protocols have shown a decline in packet delivery ratio, packet loss and packet loss ratio for both CBR and VBR traffic. For VBR traffic, AODV has hardly shown any change for PDR, PL and PL ratio whereas DSR has shown an increase in PDR and DYMO and LAR are showing a reduction in PDR. In future, we propose to consider metrics like delay and jitter on applications like FTP, Telnet and VOIP. The results of the study has indicated that by combining two or more protocols the packet loss ratio can further be reduced with improvement in PDR by mitigating error propagation in data transmission

\section{REFERENCES}

[1] Residential Broadband Internet Services and Application Requirements" T K Wok IEEE Communications Magzine June 1997.

[2] www.ciscopress.com/articles.

[3] Vinod Namboodri , L Gao IEEE Transactions 2007 “ Prediction based routing Protocol for vehicular adhoc networks

[4] Shailey Mittal "Performance comparison of AODV, DSR and ZRP routing protocols in MANETs"

[5] Mrs Vaishali and Dr ketan Katocha "Simulation based performance evaluation of routing protocols in Vehicular Adhoc Networks" International Journal of scientific and research publications Volume 3 Issue 10 Oct 2013

[6] Asma Tuteja, Rajneesh Gujral "Comparative performance analysis of DSDV,AODV and DSR in MANET using NS2

[7] N Suryati, A Abdullah and A Faisal "Performance evaluation of AODV, DSDV and DSR routing protocol in Grid environment" International Journal of Computer Science and Network Security Vol 9 No 7 July 2009

[8] Amitava Mukherjee, S Bhandyopadhyay, D Saha "Location Management and Routing in mobile Wireless Netowrks

[9] Y Be Ko, Nitin H Vaidya "Location aided routing protocol in Mobile Adhoc Networks" Wireless Networks 6 (2000) 307-321

[10] Ian D Chakeres and E M Royer AODV routing protocol implementation design. In ICDCSW '04 Proceedings of 24th international conference on distributed computing system workshops- W7 EC(ICDCSW'04) pages 698-703 Washington DC, US

[11] Ian D Chekeres and C E Perkins Dynamic Manet on Demand (DYMO) routing protocol Internet-Draft Version 06, IETF, Oct 2006 